\title{
The Quick Start Guide for Establishing a Facebook Presence for Nursing Research
}

\author{
Herbell K* \\ Case Western Reserve University, USA
}

*Corresponding author: Kayla Herbell, Case Western Reserve University, 19606 Van

Aken Blvd Apt 101 Shaker Heights, OH 44122, USA, Tel: 4405476683; E-mail:

\section{Editorial}

Volume 2 Issue 3

Received Date: April 09, 2018

Published Date: April 23, 2018

ksh57@case.edu

\section{Editorial}

Facebook represents an untapped participant pool that nurse researchers have yet to utilize to its full potential. The purpose of this article is to provide personal insights as well as data based suggestions in order to demystify Facebook recruitment. The suggestions in this article are broadly classified as: develop a trusted profile, know your target population, and craft a meaningful post. Each suggestion provides tips in order to prevent frequently observed errors in Facebook participant recruitment.

Recruitment through Facebook represents a colossal potential participant pool from which nursing researchers may elect to sample from. Between the end of 2012 and 2017, the total monthly active Facebook users (defined as users who have logged on at least once in 30 days) has increased over $47 \%$ [1]. This margin of growth has not slowed in 2018 as the total monthly users have exponentially increased every quarter since 2008 [1]. As of the end of 2017, there are an estimated 2.2 billion monthly users on Facebook alone [1].

Facebook is a popular social media modality because the platform offers several features for users. One vital feature in which researchers need to tap into is advertising/recruiting for research. The recruiting/advertising function has historically been utilized by brand agencies (i.e. ads for new soft drinks, etc.) in order to target consumers. However, there is a huge potential market of research participants that remain untapped because of the continued use of dated recruitment methods (e.g. snail mail recruitment). There may be some apprehension among nurse researchers to using Facebook recruitment because of the learning curve posed.

Therefore, the purpose of this editorial is to demystify Facebook participant recruitment by providing a comprehensive "quick start guide" of suggestions for establishing a Facebook presence. The content of this quick start guide was derived from insights from a recent social media based study as well as databased statistics on social media advertising. Below is a list of three broad suggestions derived from personal experience using Facebook as a recruitment method as well as statistics about Facebook to support these insights.

\section{Develop a Trusted Profile}

Social media can be a "faceless" platform that may not instill a sense of trust in users (i.e. potential study participants). Because of this assumption, researchers interested in recruiting with Facebook need to create a transparent and honest profile. This starts by ensuring that you have a profile photo and cover photo of people, places, or things that represent the study or the purpose of the study. For example, I have used profile and/or cover photos of my Principal Investigator or of my study's logo. Let users know that you belong to a legitimate institution and the trusted profession of nursing by also including a detailed "about me" section. The about me section should include the purpose of the research study as well as any other relevant details about the research and yourself (i.e. funding source, previous studies, a personal website for additional information). 


\section{Nursing \& Healthcare International Journal}

A second way to build trust in your profile followers is by allowing time to elapse before posting about your study that you are recruiting for. I suggest that after you complete your full profile (Facebook will walk you through this step by step) that you "follow" or "like" different profiles and establish a "friend" base. For example, if I were recruiting family caregivers, I would "like" profiles and pages associated with family care giving (e.g. the National Family Caregiver Alliance). I would also be sure to "invite" my own personal friends to "like" my study profile page. Generating "likes" and visits to your Facebook profile is important because it not only increases visibility to potential participants but also may increase the validity of your profile.

A major error that I see when working with groups who are recruiting through social media is not building that trust with a community before attempting to recruit participants. Just like in a physical community (e.g. support group) you need to build trust before advertising for your study. I suggest that you ask to join support groups on Facebook as this is a rich source of participants, however, do not advertise your study immediately upon being accepted into the group. Oftentimes there will be a gatekeeper in these private groups (i.e. a moderator). A common error I see is that new members (i.e. researchers) will be added to a private group and immediately begin posting about their study. This may cause the moderator to remove you from the group or your post may be deleted from the page. Instead, I suggest that you allow a couple of weeks to pass before posting about your study. Again, you want to build trust so consider commenting on others posts or explicitly ask (i.e. private message) the moderator of the group to post about your study.

\section{Know your Target Population}

Not every target population uses Facebook, which may be due to physical or mental capabilities or just preference. For example, younger populations may elect to use Instagram or Snapchat rather than Facebook. Different target populations also frequent their social media of choice at different times of the day. For example, $50 \%$ of users who are between the ages of 18-24 use Facebook when they wake up in the morning [2]. So if this is your target population, you will want to post in the morning to make sure these users see your ad.

If you are unable to find specific statistics on your population as to when they are the most active on Facebook these general guidelines may be useful: a) the highest mid-week traffic occurs between $1 \mathrm{pm}$ and $3 \mathrm{pm}$
[2], b) posts before $8 \mathrm{pm}$ often generate more traffic than posts after $8 \mathrm{pm}$ [2], c) Thursdays and Fridays tend to yield the highest engagement [2]. An additional tool that may be useful is Facebook Insights, a free, user-friendly analytic program that provides feedback on each of your Facebook posts. Feedback includes the amount of engagement (i.e. likes, views, etc.) per post. I have Facebook Insights to determine what type of posts (plain text vs. a poster) generate the most amount of engagement for my target audience.

\section{Craft a Meaningful Post}

One of the more harrowing lessons learned from my own work is to pay attention to what you post. Facebook has implemented an algorithm that is used to detect "bots" or profiles whose sole purpose is to spam users. The repercussion for being flagged as a bot is your profile is banned from Facebook for approximately one month meaning you cannot post, comment, or engage on Facebook at all. In order to avoid a profile being flagged as a bot make sure your posts are unique and that you do not post the same language on multiple pages. A simple way to ensure that you are not flagged as a bot is to change a couple of words in each post. For example, interchange the words "searching for participants" to "looking for individuals" or "recruiting people".

Another tip is to make sure that you are posting on your profile often. Each and every post should not be about study recruitment. If every time you post you are soliciting participants you are likely to lose followers and people whom "like" your page. Instead, create a schedule that you will follow so you stay on track of posting. For example, I post once per week soliciting research participants. Other posts are about my research topic of interest or updates about the study (e.g. how many participants enrolled to date). Anecdotally, I posted a newspaper article about a study I was working on which generated over 550 views on the study's profile page.

Researchers should also be aware of their study population prefers to view Facebook on a computer or a mobile device. There are a massive amount of Facebook mobile app users-over 1.15 billion. The frequency of mobile Facebook users illustrates the importance of ensuring that your Facebook profile is mobile app friendly, meaning, that the images displayed on your profile are high resolution and users can easily tell what the profile's purpose is. Furthermore, the average user spends about twenty minutes on Facebook per visit [2]. This is a limited amount of time in which users are being 
bombarded with information. Therefore, your post and study profile needs to be eye-catching and direct.

I suggest that you do not post paragraphs of plain textit is not attractive to users and they will likely scroll past it. Instead, consider creating a recruitment flyer that can easily be shared among users. There are nearly 300 million photo uploads to Facebook per day [2] which illustrates that sharing images is the preferred method of advertising and generating engagement on Facebook. I suggest a colorful flyer that has bullet points with simple language. When you post the flyer be sure to include a 1-2 sentence caption that encourages users to engage with the flyer by "liking" the post or "sharing" it with their friends to increase visibility.

In conclusion, with the exponential growth of Facebook users, Facebook still remains a relatively untapped market for participant recruitment into nursing research. Nurse researchers interested in using this innovative modality should follow the steps above to curtail some of the apprehension around using this new recruitment method. These useful and simple suggestions: developing a trusted profile, knowing your target population, and crafting a meaningful post are all valuable suggestions that may enhance a researcher's Facebook presence and entice participants.

\section{References}

1. Facebook users worldwide 2017 (n.d.).

2. Noyes D (2018) The top 20 valuable Facebook statistics-updated April 2018. 